BNL-NCS-51800

(Rev. 03/07)

Formal Report

\title{
NUCLEAR SCIENCE REFERENCES
} CODING MANUAL

\author{
David F. Winchell
}

March 20, 2007

INFORMATION ANALYSIS CENTER REPORT

NATIONAL NUCLEAR DATA CENTER BROOKHAVEN NATIONAL LABORATORY UPTON, LONG ISLAND, NEW YORK 11973 


\section{DISCLAIMER}

This work was prepared as an account of work sponsored by an agency of the United States Government. Neither the United States Government nor any agency thereof, nor any of their employees, nor any of their contractors, subcontractors or their employees, makes any warranty, express or implied, or assumes any legal liability or responsibility for the accuracy, completeness, or any third party's use or the results of such use of any information, apparatus, product, or process disclosed, or represents that its use would not infringe privately owned rights. Reference herein to any specific commercial product, process, or service by trade name, trademark, manufacturer, or otherwise, does not necessarily constitute or imply its endorsement, recommendation, or favoring by the United States Government or any agency thereof or its contractors or subcontractors. The views and opinions of authors expressed herein do not necessarily state or reflect those of the United States Government or any agency thereof. 
BNL-NCS-51800

(Rev. 03/07), UC-34C

(Physics-Nuclear - TIC-4500)

Formal Report

\title{
NUCLEAR SCIENCE REFERENCES CODING MANUAL
}

\author{
David F. Winchell
}

March 20, 2007

\section{INFORMATION ANALYSIS CENTER REPORT}

Notice: This manuscript has been authored by employees of Brookhaven Science Associates, LLC under Contract No. DE-AC02-98CH10886 with the U.S. Department of Energy. The publisher by accepting the manuscript for publication acknowledges that the United States Government retains a non-exclusive, paid-up, irrevocable, world-wide license to publish or reproduce the published form of this manuscript, or allow others to do so, for United States Government purposes. 


\section{Contents}

1 Introduction 1

2 Structure of the NSR File 3

2.1 KEYNO . . . . . . . . . . . . . . . . . . 4

2.2 HISTORY . . . . . . . . . . . . . . . . 4

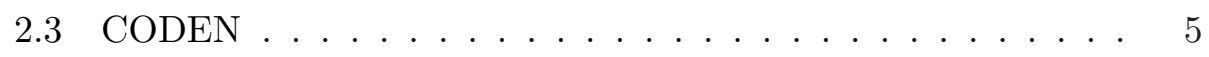

2.3 .1 JOUR ...................... 6

$2.3 .2 \mathrm{CONF} \ldots \ldots \ldots \ldots \ldots$

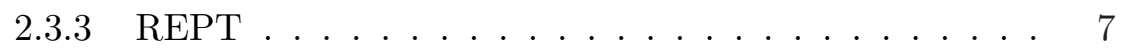

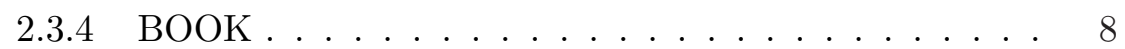

$2.3 .5 \mathrm{PC} \ldots \ldots \ldots \ldots \ldots \ldots$

2.3 .6 THESIS . . . . . . . . . . . . . . . 9

2.3 .7 PREPRINT . . . . . . . . . . . . 9

2.4 REFRENCE . . . . . . . . . . . . . . . . . . 9

2.4 .1 JOUR . . . . . . . . . . . . . . . . 10

$2.4 \mathrm{CONF} \ldots \ldots \ldots \ldots 11$

$2.4 .3 \mathrm{REPT} \ldots \ldots \ldots \ldots \ldots 11$

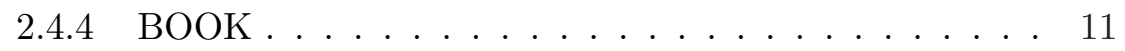

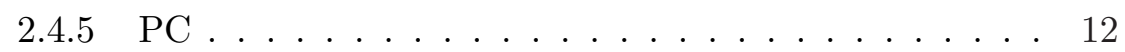

2.4 .6 THESIS . . . . . . . . . . . . . . . . 12

2.4 .7 PREPRINT . . . . . . . . . . . . . . . 12

2.5 AUTHORS . . . . . . . . . . . . . . . 12

2.6 TITLE . . . . . . . . . . . . . . . . . . 13

2.7 KEYWORDS . . . . . . . . . . . . . . 13 
2.8 SELECTORS . . . . . . . . . . . . . . . . . . . . . 14

2.9 DOI . . . . . . . . . . . . . . . . . . 14

3 Keyword Abstracts $\quad 15$

3.1 Structure of keyword abstracts . . . . . . . . . . . 15

3.2 Inclusion criteria and coding procedures for specific topics . . 18

3.2.1 NUCLEAR REACTIONS . . . . . . . . . . . . . . 19

3.2 .2 RADIOACTIVITY . . . . . . . . . . . 20

3.2 .3 NUCLEAR STRUCTURE . . . . . . . . . . 21

3.2 .4 NUCLEAR MOMENTS . . . . . . . . . . . . 21

3.2 .5 COMPILATION . . . . . . . . . . . . . . . . 21

3.2 .6 ATOMIC MASSES . . . . . . . . . . . . . 21

3.2 .7 ATOMIC PHYSICS . . . . . . . . . . . . . 21

3.2.8 MULTIPLE TOPICS . . . . . . . . . . . . 21

3.3 Selectors . . . . . . . . . . . . . . . . . . 22

3.4 General Considerations and Conventions . . . . . . . . . . . 23

4 Scope and Completeness $\quad 27$

$\begin{array}{lr}\text { A Journals and Codens } & 29\end{array}$

B Abbreviations 31

$\begin{array}{ll}\text { C Special Characters } & 33\end{array}$ 


\section{Chapter 1}

\section{Introduction}

This manual is intended as a guide for Nuclear Science References (NSR) compilers. The basic conventions followed at the National Nuclear Data Center (NNDC), which are compatible with the maintenance and updating of and retrieval from the Nuclear Science References (NSR) file, are outlined.

The NSR database originated at the Nuclear Data Project (NDP) at Oak Ridge National Laboratory as part of a project for systematic evaluation of nuclear structure data. ${ }^{1}$ Each entry in this computer file corresponds to a bibliographic reference that is uniquely identified by a Keynumber and is describable by a Topic and Keywords. It has been used since 1969 to produce bibliographic citations for evaluations published in Nuclear Data Sheets. Periodic additions to the file were published as the "Recent References" issues of Nuclear Data Sheets prior to 2005. In October 1980, the maintenance and updating of the NSR file became the responsibility of the NNDC at Brookhaven National Laboratory. The basic structure and contents of the NSR file remained unchanged during the transfer.

In Chapter 2, the elements of the NSR file such as the valid record identifiers, record contents, and text fields are enumerated. Relevant comments regarding a new entry into the NSR file and assignment of a keynumber are also given in Chapter 2. In Chapter 3, the format for keyword abstracts is given followed by specific examples; for each TOPIC, the criteria for inclusion of an article as an entry into the NSR file as well as coding procedures are described. Authors preparing Keyword abstracts either to be published in a Journal (e.g., Nucl. Phys. A) or to be sent directly to NNDC (e.g., Phys. Rev. C) should follow the illustrations in Chapter 3. The scope of

\footnotetext{
${ }^{1}$ See W.B.Ewbank, ORNL-5397 (1978).
} 
the literature covered at the NNDC, the categorization into Primary and Secondary sources, etc., is discussed in Chapter 4. Useful information regarding permitted character sets, recommended abbreviations, etc., is given in the Appendices.

The NSR database has been in existence for decades, and responsibility for its upkeep has passed through many hands. Those familiar with the contents of NSR will note that not all of the formats and conventions discussed in this manual have always been adhered to. In recent years, however, these conventions have been followed fairly consistently, and it is expected that the preparation of new entries will follow these guidelines.

The most up-to-date information about NSR contents and policies can be found at the NSR web site: http://www.nndc.bnl.gov/nsr.

This manual is an update to BNL-NCS-51800 (Rev. 08/96) by S. Ramavataram and C.L. Dunford. Discussions with Mark Kellett of the IAEA are gratefully acknowledged, as are comments and suggestions from the NNDC staff and members of the U.S. Nuclear Data Program. This manuscript has been authored by Brookhaven Science Associates, LLC under Contract No. DE-AC02-98CH1-886 with the U.S.Department of Energy. 


\section{Chapter 2}

\section{Structure of the NSR File}

The NSR "exchange format" file uses an ASCII character format of logical records initiated by a ten-character identifier. In the standard format, the file contains 80-character physical records with the start of each logical record coinciding with the beginning of a physical record. In some instances, an ampersand (\&) character is added in the 81st column to indicate the end of the line. (Because the ampersand is used to indicate end-of-line, it should never be used within a record; the spelled-out word "and" should be used in its place.)

The legal record identifiers are:

$<$ KEYNO $>$ - Reference keynumber

$<$ HISTORY $>-$ Administrative record

$<$ CODEN $>$ - Standard form reference

$<$ REFRENCE $>$ - Free text reference

$<$ AUTHORS $>-$ Author names

$<$ TITLE $>$ - Reference title

$<$ KEYWORDS $>-$ Keyword abstract

$<$ SELECTRS $>$ - Indexing parameter list

$<$ DOI $\quad>$ - Digital object identifier

Only these record types appear in the file and they appear in the above order. Only one record of each type appears in the file for each reference, except for <KEYWORDS $>$ and <SELECTRS $>$, which will be repeated for each major category (i.e., NUCLEAR REACTIONS, NUCLEAR STRUCTURE, etc.) under which the reference is indexed. 
It should be noted that the $<$ KEYNO $>$ and $<$ HISTORY $>$ fields are modified when a new file is merged with the database. Both the pre- and postmerge formats are described below.

\section{$2.1 \quad \mathrm{KEYNO}$}

Each entry in the database has a unique eight-character keynumber. The first four characters are the publication year, the next two are the first two letters of the first author's last name, and the final two are a unique identifier (2 digits for a Primary reference or 2 letters for a Secondary reference). The keynumbers are upper case as shown in example below.

1976LA03 - Primary

1976LAZY - Secondary

The last two characters in the keynumber are assigned when the entry is added to the database. Historically, there have been three two-letter combinations for which NSR "runs out" of keynumbers in a given year (that is, there are more than 99 primary references where the first author's name starts with those two letters). Those cases are "BA", "BE", and "MA". When NSR runs into the 100th case of these in a given year, the letter combinations "BB", "BF", and "MB" are used instead.

For an initial entry prepared in the "compiled" mode (see section 2.2) the keynumber will have seven characters. The first six have the same significance as above. The last character is $\mathrm{P}$ for Primary source, $\mathrm{S}$ for Secondary source. Thus, in the update input file the record would be:

$<$ KEYNO >1976LAP

or

<KEYNO >1976LAS

\subsection{HISTORY}

Once an entry has been added to the database, the history field will contain the letter "A" (added) followed by a date in the form YYYYMMDD indicating the date that the reference was added to the database. In addition, 
there can be a second field consisting of "M" (modified) followed by the most recent modification date. For example:

<HISTORY >A20020523

or

<HISTORY >A20031215 M20040125

For files to be entered into the database, this record contains a single character code "C" or "M" followed by a date in the form YYYYMMDD. C (compiled) should be used for new entries with seven-character keynumbers (see above), M (modified) should be used for modifications of existing entries, and so the corresponding eight-character keynumber should be given:

$<$ KEYNO >2005CAP

$<$ HISTORY >C20050425

or

$<$ KEYNO >2004BA02

$<$ HISTORY >M20050425

The actual date that the file is added to the database will be inserted into the history field at that time (preceded by A or M).

Note: In the past, there was a "D" (deleted) code for the History field. This is no longer used. If an entry is invalid for some reason (it is a duplicate, or the publication year is wrong, for instance) then a minimal entry is given ( $<$ KEYNO $>,<$ HISTORY $>,<$ CODEN $>$ ) along with a pointer to the correct entry. The reason for this is that once a keynumber is "published" in the database, it might be used in the literature, and so it should be persistent in the database.

\subsection{CODEN}

This field consists of a reference-type code followed by an abbreviated form of the reference. This field cannot have more than 70 characters (80 characters total including the $<$ CODEN $>$ tag). The field should always begin with one of the permitted reference types: 


$\begin{array}{ll}\text { JOUR } & \text { - Journal } \\ \text { CONF } & \text { - Conference } \\ \text { REPT } & \text { - Report } \\ \text { BOOK } & \text { - Book } \\ \text { PC } & \text { - Private Communication } \\ \text { THESIS } & \text { - Thesis } \\ \text { PREPRINT } & \text { - Preprint }\end{array}$

\subsubsection{JOUR}

For Primary sources (Journals) the $<$ CODEN $>$ format is standard. A list of Journals scanned at NNDC together with the approved five-letter CODEN values is attached (Appendix A).

A standard <CODEN $>$ field for a journal will include the five-letter "Coden" abbreviation for the journal, followed by the volume and page number, separated by spaces:

JOUR [Coden] [Vol] [Page]

Example:

<CODEN > JOUR NUPAB 750185

This represents an article from Nuclear Physics A, volume 750, page 185. For journals such as Physical Review that assign article numbers rather than page numbers, the article number should be used.

In the (rare) cases where page numbering is done by issue rather than volume, the issue number should be given:

<CODEN > JOUR RNCUA 26 No3,1

In addition to regular journal articles, abstracts of Physical Society meetings and Thesis abstracts have been coded under this category. The format is similar to the one given above, with the addition of the first author's last name at the end (and the abstract number for BAPSA abstracts):

JOUR [coden] [vol] [page],[abstract no.],[First Author's Last Name]

<CODEN >JOUR BAPSA 18 720,KK12, Nawrocki

<CODEN > JOUR DABBB 41 4162, Liu 


\subsubsection{CONF}

At minimum, the $<$ CODEN $>$ field for an article from a conference proceedings should include the conference location and the page number of the article. It is also often helpful to include the subject of the conference (usually a shortened version of the conference title) and the first author's last name.

CONF [Location]([Subject]),P[page number],[First Author's last name]

Example:

<CODEN >CONF Studsvik (n, Gamma Spectroscopy), P403, Bohm

The author's name may be left off if it will not fit in the allowed 70character space.

If the Reference was from contributed abstracts published separately from full papers, identify by placing "Contrib" before the page number:

<CODEN >CONF Tokyo(Nuclear Structure) Contrib,P403,Bohm

In some cases, the optional identification "Proc" is used to distinguish the reference from a "contributed" volume. This is shown in the example below:

<CODEN >CONF Santa Fe (Nucl Data for Sci and Technol) Proc,Vol1,P688

The above example also illustrates how multi-volume sources are coded. The volume number appears immediately before the page specification.

\subsubsection{REPT}

These are generally numbered laboratory reports or annual reports.

REPT [rept-number],P[page number],[First Author's last name]

Example:

<CODEN >REPT INDC (CCP) -164/1, P9, Skarastov

If the entry is for the entire report, the page number may be omitted. 
<CODEN >REPT JINR-E1-2005-17,Bellettini

For references from laboratory annual reports without specific code number:

<CODEN >REPT Univ Arkansas Nucl Chem Ann Rept,P4, Kantele

\subsubsection{BOOK}

The book title and publisher should be provided, within the 70-character constraint:

BOOK [Title/Abbreviated Title], [Publisher]

Example:

<CODEN >BOOK Table of Isotopes. 7th Ed., John Wiley and Sons

If the reference refers to an article within a book, the page number should also be provided. In addition, volume numbers should be given for multivolume titles, and the first author's name should be included if space permits:

<CODEN >BOOK Particle Emission from Nuclei, Vol3,P99, CRC Press, Hardy

\subsubsection{PC}

Private communications are typically entered at the request of evaluators. Copies of the communications received since 1981 are archived at the NNDC.

PC First Author's initials separated by 1 space followed by last name

Example:

$<$ CODEN >PC E Spejewski

If Private Communication was dated, add date after author name:

<CODEN >PC E Spejewski,5/28/80 


\subsubsection{THESIS}

$\mathrm{PhD}$ theses are identified by author and institution:

THESIS Author's initials separated by 1 space followed by last name,Affiliation

Example:

<CODEN > THESIS W Roney, Univ New Mexico

If the thesis abstract is published in Dissertation Abstracts:

<CODEN >THESIS W Roney, Univ New Mexico,DABBB 322339

\subsubsection{PREPRINT}

Preprints from laboratories often have an associated report number:

<CODEN >PREPRINT PPP/4-8 1, Ajzenberg-Selove

If preprint has no report number, author's name and date should be given:

<CODEN >PREPRINT Ajzenberg-Selove,4/20/1990

Articles from the arxiv.org e-print archive are included as preprints in NSR:

<CODEN >PREPRINT nucl-ex/0503021,3/29/2005

\subsection{REFRENCE}

This is a free text field, and can exceed 70 characters. In other words, the logical record can span more than one 80-character line in the exchangeformat file. It should always contain the publication year of the reference, in parentheses. (This should match the publication year indicated in the keynumber). 


\subsubsection{JOUR}

The $<$ REFRENCE $>$ field for journal sources is standardized.

[journal title] [volume], [page] ([year])

Example:

<REFRENCE>Phys.Rev. C18, 424 (1980)

If an Erratum or Comment is published for this Reference in a subsequent issue of the Journal, the entry will be modified as:

<REFRENCE>Phys.Rev. C18, 424 (1980); Erratum Phys.Rev. C20,678 (1981)

or

<REFRENCE>Phys.Rev . C18, 424 (1980); Comment Phys.Rev. C20,678 (1981)

For journals that begin page numbering with each issue rather than each volume, the issue number should be provided:

<REFRENCE>Riv. Nuovo Cimento Soc.Ital.Fis. 26, No 3, 1 (2003)

Russian Journals for which English translations are available are a special case. In the past, the Russian information was given first, followed by information from the translated journal:

<REFRENCE>Yad.Fiz. 68, 1407 (2005); Phys.Atomic Nuclei 68, 1352 (2005)

Beginning in 2006, the articles in Phys.Atomic Nuclei no longer consistently included information on the original Russian publication. In this case, we have switched the order and are putting the translation journal first, and the Russian information second, where available:

<REFRENCE>Phys.Atomic Nuclei 69, 197 (2006); Yad.Fiz. 69, 219 (2006)

Note that the <CODEN $>$ field should, in either case, use the volume and page number of the journal that appears first in the reference field, and the keynumber should reflect the publication year for that journal. For other journals appearing in both original and translated form, decisions will be made on a case-by-case basis at the NNDC. 


\subsubsection{CONF}

The information should include the conference title and location, along with page number and publication year. In addition, conference dates and proceedings editors may be indicated.

<REFRENCE>Intern. Conf. Neutron Capture Gamma-Ray Spectroscopy, Studsvik, p.403 (1969)

<REFRENCE>Proc. XLII Intern. Winter Meeting on Nuclear Physics, Bormio, Italy, 25 - 31 January 2004, I.Iori ed., p.315 (2004)

If the reference is from a volume of contributed abstracts, substitute "Contrib." for "Proc.".

When the conference is part of a series, such as the AIP conference proceedings, that information should be added at the end of the field:

$<$ REFRENCE>Proc.Nuclei at the Limits, Argonne, Illinois, D.Seweryniak and

T.L.Khoo, eds., p.93 (2005); AIP Conf. Proc 764 (2005)

\subsubsection{REPT}

If a Report has number assigned:

<REFRENCE>NDC (CCP) - 1 64/L, p.9 (1981)

If Reference is from an annual lab report without specific report number:

<REFRENCE>Univ.Arkansas, Nucl.Chem.Res., Ann.Rept., p.4 (1963)

\subsubsection{BOOK}

[Title], [Publisher], [Address] ([year])

Example:

<REFRENCE>Table of Isotopes, 7th Ed., John Wiley and Sons, Inc., New York (1978)

For multivolume titles, the volume number should be included. For articles within books, page numbers should be provided:

<REFRENCE>Particle Emission from Nuclei, Vol.3, p.99, CRC Press, Florida (1989) 


\subsubsection{PC}

The reference field for private communications is always the same:

$<$ REFRENCE >Priv.Comm . (1980)

\subsubsection{THESIS}

Thesis, [Affiliation] ([year])

Example:

<REFRENCE>Thesis, Univ.New Mexico (1969)

If Thesis abstract is published in Dissertation Abstracts

<REFRENCE>Thesis, Univ.New Mexico (1969); Diss.Abst.Int. 32B, 2229 (1969)

\subsubsection{PREPRINT}

If PREPRINT has a report number:

[report number] $([$ year $])$

Example:

$<$ REFRENCE $>$ PPP/4-81 (1981)

If there is no report number:

<REFRENCE>Priv.Comm. (1981)

For articles from the arxiv.org e-print archive:

<REFRENCE>nucl-ex/0503021,3/29/2005（2005）

\section{$2.5 \quad$ AUTHORS}

This is a free-text field. Author names should be separated by commas. First and middle names, if given, should be replaced by initials. There should be 
a space after each comma, but no spaces following the "." after an initial. Only the last name and first initial are indexed within the database. If a collaboration name is given, it should be appended to the author list in the form ", and the XXX Collaboration". If there are more than 35 authors, and a collaboration is given, it is permissible to list only the first author's name and the collaboration. (In the database the "first initial" for the collaboration is indexed as "+"). Examples:

<AUTHORS >P.Oblozinsky, M.Herman, S.Mughabghab, I.Sirakov, J.Chang, T.Nakagawa, K.Shibata, M.Kawai, A.V.Ignatyuk, V.G.Pronyaev, V.Zerkin, Q.Shen, Y.Zhuang

$<$ AUTHORS >S.S.Adler, and the PHENIX Collaboration

\subsection{TITLE}

This is also a free-text field. It should contain the title of the reference. For symbols such as greek characters, and for super- and sub-scripted characters, the substitutions outlined in Appendix $\mathrm{C}$ should be used. Examples:

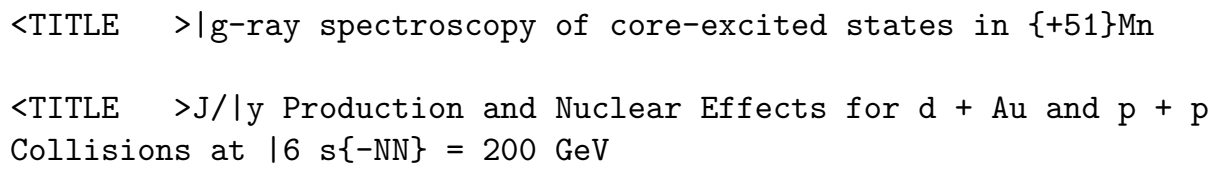

\subsection{KEYWORDS}

Because the keywords in NSR are indexed using a computer program, they need to follow fairly strict formatting rules. Each keyword abstract consists of a major topic, followed by one or more indexed sentences, followed by zero or more non-indexed sentences. A given reference may have more than one keyword abstract, if more than one major topic is relevant. There should be only one set of keywords per major topic. In the NSR exchange format, each keyword abstract is preceded by a $<$ KEYWORDS $>$ tag. Details on keyword structure and contents are given in the next chapter.

It should be noted that, for certain types of articles, an NSR entry might not have any keywords. Because the goal of the keywording is to index specific physical systems and related quantities, articles such as reviews or general theoretical papers may not have content that lends itself to the 
production of meaningful keyword abstracts. These papers are still entered into the database if they fall within the scope of NSR, and can generally be retrieved by author or by text searches on the title. (During the ten-year period 1996-2005, keyword abstracts were prepared for approximately $74 \%$ of entries, the rest were entered without keywords).

\subsection{SELECTORS}

The "selector" field is generated by computer, using a pre-processing program, based on the content of the keyword abstract. It is used to index the entry in the database. There will be one $<$ SELECTRS $>$ field for each $<$ KEYWORDS $>$ field. These fields are alway generated before adding the entry to the database; the entry will be rejected by the loading program if it contains $<$ KEYWORDS $>$ fields without associated $<$ SELECTRS $>$ fields. The person preparing the keywords should keep selector generation in mind. When feasible, the pre-proccessing program should be run as part of the proofreading process. Examples of generated selectors will be discussed in the next chapter. Selectors will be discussed further in Section 3.3.

\subsection{DOI}

A "DOI", or digital object identifier, is a code assigned by the publisher which allows uniform access to articles via the World Wide Web. In webbased dissemintation of NSR entries, this can be used to link the the article in question. When an article is published with a DOI, the field is added at the same time as the reference, author, and title information. In some instances, the publisher assigns a DOI but does not include the information in the published paper. In those cases, the NNDC will attempt to determine the DOI, typically by using the crossref .org service. More information on digital object identifiers can be found at http://www.doi.org/. 


\section{Chapter 3}

\section{Keyword Abstracts}

What distinguishes NSR from more general bibliographic databases is the level of detail provided in the keyword abstracts. In preparing the keyword abstract, therefore, it is important to pick out the specific physical systems being studied (isotopes, reactions, etc.), and the quantities being discussed (i.e. measured cross sections, calculated energy levels ...). A great deal of the required information can often be extracted from the abstract, figures, tables, and conclusions.

It is worthwhile keeping in mind that the keywords serve a dual purpose. First, they are used to generate "selectors", as described above, which are then used to index the article for search purposes. Searching can also be done on the text of the keywords. Second, when a user retrieves a list of entries, the keywords can help him or her determine whether the paper is of interest.

Because of the variety of papers covered in NSR, there is some flexibility in how a given paper is keyworded. On the other hand, it is important to maintain as much consistency as possible in order for the indexing to be a useful tool. It is suggested that keyword preparers consult recent entries for similar papers when questions arise as to specific formats and phrasing.

\subsection{Structure of keyword abstracts}

Schematically, a keyword abstract will look like the following (items in parenthesis are optional, and "..." indicates that more than one element of the type may be present): 
$<$ KEYWORDS $>$ [Major topic] [Indexed sentence] ([Indexed sentence] ...)

([non-indexed sentence] ...)

The various keyword elements are discussed in the following:

[Major topic]: The following major topics are allowed - NUCLEAR REACTIONS, RADIOACTIVITY, NUCLEAR STRUCTURE, NUCLEAR MOMENTS, ATOMIC PHYSICS, ATOMIC MASSES, and COMPILATION. Most experimental articles will fall in the first two categories. The scope of each topic will be discussed in the next section.

Note that, in the past, major topics were sometimes followed by a "minor topic". This is being phased out.

[Indexed sentence]: Sentences to be indexed have a specific format in NSR. Each sentence consists of two or more phrases separated by semicolons (;) and ending with a period (.). The first phrase describes the physical system (nuclides, reactions, etc), and at lease one subsequent phrase should describe measured/deduced/calculated quantities:

[System]; ([System]; ...) [Quantities] (; [Quantities] ...).

[System] phrases: There are three types of "System" phrase: nuclide list, reaction list, and decay list. A single sentence can have one or more system phrases at the beginning; if there is more than one, they should all be the same type.

A nuclide list can be a single isotope or a comma-separated list of isotopes. (In this context, "isotope" can also refer to a chemical symbol without a mass number.)

Single isotopes :

$\mathrm{Ca}$

$\{+40\} \mathrm{Ca}$

Isotope lists:

$\{+40\},\{+44\} \mathrm{Ca}$

$\{+208\} \mathrm{Pb},\{+209\} \mathrm{Bi}$

$\{+4\},\{+5\},\{+6\} \mathrm{He},\{+7\},\{+8\} \mathrm{Li}$ 
In some cases, an "isotope list" can be in the form " $\mathrm{A}=$ num" or " $\mathrm{Z}=$ num" where "num" is a number or range of numbers in the form " $a-b$ ". This is generally only used when the authors are compiling or calculating quantities for a large number of nuclides (100 or more), and listing individual nuclides would be impractical. It is also worth noting that listing a very large number of individual isotopes could be detrimental in search applications, as one could find a situation where the same few articles (with many nuclides listed) showing up in every nuclide search.

Reaction lists consist of one or more targets, followed by one or more reactions in the form (in,out), followed by an energy specification. Target lists have the same form as nuclide lists. Residual nuclides are generally not given if they can be calculated. Otherwise, residuals can be optionally inserted between the reaction and energy as a "/" delimited list. Examples:

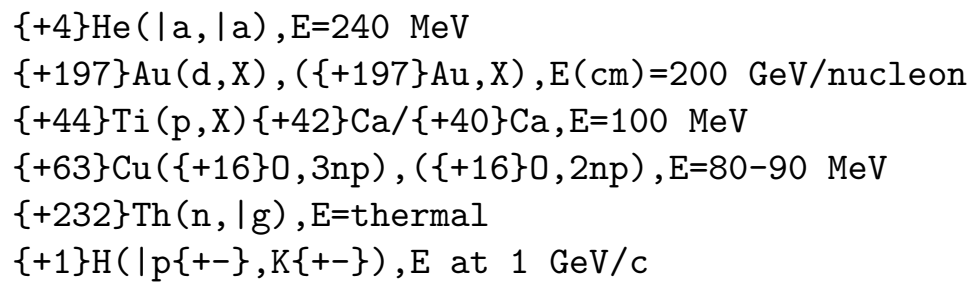

Note that "E" by itself denotes lab energy. More details regarding energy specification are given in the section 3.2.

For fixed-target experimental papers where the system information is going to be followed by a "measured" phrase, the target should always be given as it is in the laboratory frame of reference, even if the paper goes on to analyze the results in "inverse kinematics". Additional sentences may give deduced quantities for the inverse reaction.

Decay lists consist of isotope lists (parent nuclides) followed by one or more decay modes in parentheses:

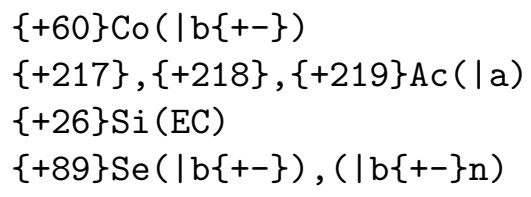

When the origin of the unstable nuclide is described in the paper, it should be given in square brackets (note, however, that the information in the brackets is not indexed): 
$\{+225\}$ Ac ( | a $) \quad[$ from $\{+226\} \operatorname{Ra}(\mathrm{p}, 2 \mathrm{n})]$

$\{+116 \mathrm{~m}\} \operatorname{Ag}(\mathrm{IT}),\{+116\} \operatorname{Ag}(\mid \mathrm{b}\{+-\})$ [from $U(p, F)]$

As a rule the [System] phrase or phrases in the first sentence of the keyword abstract should match the major topic. That is, NUCLEAR REACTIONS should be followed by a reaction list, and RADIOACTIVITY should be followed by a decay list.

[Quantity] phrases: These phrases begin with an indication of the type of quantity, such as "measured", "deduced", or "analyzed", followed by a list of quantities. This list will be parsed by the selector generation program to create items for the subject index. The keyword preparer has some leeway in determining how to classify various quantities. For "measured" to be used, the paper should be reporting measurements made by the authors, and give some details regarding the experimental set-up. As a rule, "deduced" quantities follow some other type, such as measured, analyzed, or calculated.

Special case for quantity phrases - If the sentence is describing deduced properties of a nuclide or list of nuclides from an experimental paper, the semicolon following the nuclide list is optional, and "deduced" need not be the first word in the quantity phrase, if an alternative formulation is clearer:

$\{+76\} \mathrm{Kr}$ deduced levels, J, | p.

$\{+145\} \mathrm{Pm}$ level deduced $\mathrm{T}\{-1 / 2\}$.

[non-indexed sentence]: In many cases, it is worth adding an additional sentence or two about the experimental or theoretical techniques used in the paper. These should be fairly succinct; the idea is to let the user know whether the paper is of interest, as opposed to giving every detail. It is also worth keeping in mind that, while this information will not be indexed, it can be accessed via a "plain-text" search. By tradition, if a "major" detector array such as GAMMASPHERE or EuROGAM is used, it is mentioned in a non-indexed sentence.

\subsection{Inclusion criteria and coding procedures for specific topics}

Following are inclusion criteria and specific keywording considerations for the various Major Topics. In general, most experimental papers are coded as NUCLEAR REACTIONS or RADIOACTIVITY. 


\subsubsection{NUCLEAR REACTIONS}

Inclusion Criteria: Hadron, light-, heavy-ion, electron, photon, meson induced reactions are included. Target should be a nucleus or nucleon.

For fixed-target experiments (i.e., not colliders), the NSR entry should consider the target to be the nuclides at rest in the laboratory frame, even if the article is reporting in the frame of reference of the "inverse reaction". For example, if the authors report "proton scattering from ${ }^{6} \mathrm{He}$ ", and the experiment involves a fixed hydrogen target, the NSR keywords should be written as $\{+1\} \mathrm{H}(\{+6\} \mathrm{He},\{+6\} \mathrm{He})$ or $\{+1\} \mathrm{H}(\{+6\} \mathrm{He}, \mathrm{p})$.

As discussed in the previous section, the "system" phrase for a reaction keyword sentence should include a target or targets, one or more reaction strings in the form "(A,B)", and a projectile energy specification. Projectile energy can be specified in any of the following ways:

- $\mathrm{E}=---\mathrm{MeV}$; implies projectile energy was specified in the article in the laboratory frame.

- $\mathrm{E}(\mathrm{cm})=---\mathrm{MeV}$; this is used when the researchers have quoted projectile energy in center-of-mass system.

- $\mathrm{E}=---\mathrm{MeV} / \mathrm{nucleon}$; this is used for heavy-ion reactions when in the article the laboratory energy is not specified anywhere.

- $\mathrm{E}$ at --- MeV/c; this notation is used when projectile momentum is specified.

- E at rest; used for reactions where the projectile is stopped in target.

- $\mathrm{E} \mid$ ? --- MeV; can be used when energy or range is only approximately defined in article.

- E |? threshold; can be used when energy is only described as being "near threshold".

- $\mathrm{E}\{+*\}=---\mathrm{MeV}$; only excitation energy is given in article.

- E=high; can be used at compiler's discretion, if energy is not specified but is clearly on the order of several GeV.

- E not given; used when the projectile energy is not specified anywhere in the article.

In general, one should use the same units as the article, "MeV" are used here for the sake of example. Energies may be single numbers, a series of comma-separated numbers, or a range specified by two numbers separated by "-". Note that |? translates to "approximately equal" (see appendix C).

For neutron-induced reactions the following can be used: 
- $E$ = reactor spectrum;

- $\mathrm{E}=$ low;

- $\mathrm{E}=$ slow;

- $E$ = fast;

- $E$ = thermal;

When properties of residual nuclides are deduced from the reaction, this information should be included in a separate sentence. Schematically, this would look like:

[reaction phrase]; [measured quantities]. [nuclide phrase] [deduced quantities].

For example:

$<$ KEYWORDS >NUCLEAR REACTIONS $\{+58\} \mathrm{Ni}(\{+29\} \mathrm{Si}, 2 \mathrm{p} 2 \mid \mathrm{a}), \mathrm{E}=128 \mathrm{MeV}$; measured $\mathrm{E}|\mathrm{g}, \mathrm{I}| \mathrm{g},|\mathrm{g}| \mathrm{g}-$, (charged particle) $\mid \mathrm{g}$-coin. $\{+77\} \mathrm{Kr}$ deduced high-spin levels, J, $\mid \mathrm{p}$, configurations .

Note that "coincidence" measurements are typically described in a pairwise fashion, even when triple or higher-fold coincidences are described in the article.

\subsubsection{RADIOACTIVITY}

Inclusion Criteria: $\beta-, 2 \beta-, \epsilon-, \alpha$-decay, delayed-particle emission, spontaneous fission, exotic decay related information is included. For a given parent nuclide, all possible decay modes are specified. If there is a string of nuclei, all nuclei with a given decay mode can be grouped together and the decay mode specified after the last nuclide. This will result in proper indexing and economy of indexing variables generated in the <SELECTRS $>$ string. If properties of daughter nuclides are deduced, these should be described in a separate sentence.

For example:

$<$ KEYWORDS>RADIOACTIVITY $\{+118\} \mathrm{Rh}(\mid \mathrm{b}\{+-\})$ [from U $(\mathrm{p}, \mathrm{F})]$; measured Elg, I|g, |b|g-coin. $\{+118\}$ Pd deduced levels, J,|p. Level systematics in neighboring isotopes discussed. 


\subsubsection{NUCLEAR STRUCTURE}

Inclusion Criteria: Any model calculation of levels, electromagnetic transition probabilities, multipole moments, nuclear form factors, giant resonance characteristics, nuclear systematics, binding energies, etc. An analysis of systematics from published data would also be included under this topic. As a rule, papers which describe new experimental results will not be keyworded under this topic.

\subsubsection{NUCLEAR MOMENTS}

Inclusion Criteria: Hyperfine structure, isotope shift, mesic X-rays, isomer shift information. Both experimental and theoretical articles are coded. Format will generally be similar to structure or radioactivity keywords.

\subsubsection{COMPILATION}

Inclusion Criteria: Any evaluation or compilation of nuclear data. All $\mathrm{Nu}$ clear Data Sheets and most Atomic Data and Nuclear Data Table articles fall under this category.

\subsubsection{ATOMIC MASSES}

Inclusion Criteria: Papers in which atomic masses are measured "directly", such as Penning trap measurements.

\subsubsection{ATOMIC PHYSICS}

Inclusion Criteria: Mesic atoms, charged-particle induced X-ray emission, etc. Format will be similar to reaction or structure keywords.

\subsubsection{MULTIPLE TOPICS}

In some cases, it is appropriate to include keyword abstracts for more than one topic. For instance, a paper might present nuclear structure information from reaction data, and also have extensive calculations of nuclear structure properties. In this case, having both NUCLEAR REACTIONS and NUCLEAR STRUCTURE keywords would be appropriate. Another common situation occurs when a reaction is studied, and the decay properties of residual radioactive nuclides are discussed. For example: 
<KEYWORDS >NUCLEAR REACTIONS $\{+144\} \operatorname{Sm}(\{+51\} \mathrm{V}, \mathrm{X}), \mathrm{E}=230 \mathrm{MeV}$; measured E|g, E $\mid \mathrm{a}$, (recoil)|a-,|a|a-,|a|g-coin following residual nucleus decay; deduced evidence for $\{+192\}$ At.

$<$ KEYWORDS $>$ RADIOACTIVITY $\{+192\},\{+192 \mathrm{~m}\}$ At $(\mid \mathrm{a})$ [from $\{+144\} \operatorname{Sm}(\{+51\} \mathrm{V}, 3 \mathrm{n})]$; measured $\mathrm{E}|\mathrm{a}, \mathrm{E}| \mathrm{g},|\mathrm{a}| \mathrm{g}$-coin, $\mathrm{T}\{-1 / 2\}$; deduced isomeric states energies, configurations.

\subsection{Selectors}

Selectors are generated from keyword abstracts to provide a consistent and coherent basis for indexing entries. While the keyword preparer is not directly responsible for creating selectors, it is useful to understand how they are generated and what the format is. Among other things, looking at selectors can be an aid to proofreading keyword abstracts.

Selectors are given as a list of elements in the form [type]:[value]; [subkey]. The [type] is a single letter describing the selector type. It should be be one of N, T, R, S, M, D, C, X, A, or Z, which stand for nuclide, target, reaction, subject, measured, deduced, calculated, other subject, mass range, and charge range, respectively. The "other subject" type is applied when a quantity phrase starts with something besides "measured", "deduced", or "calculated". If a quantity phrase contains no recognized words, a selector string with the value "OTHER" is inserted as a placeholder to indicate the existence of the phrase in the keyword abstract. The [subkey] is again a single letter, used to tie together selectors from the same sentence.

The example below repeats a keyword example from the previous section, this time with selectors included:

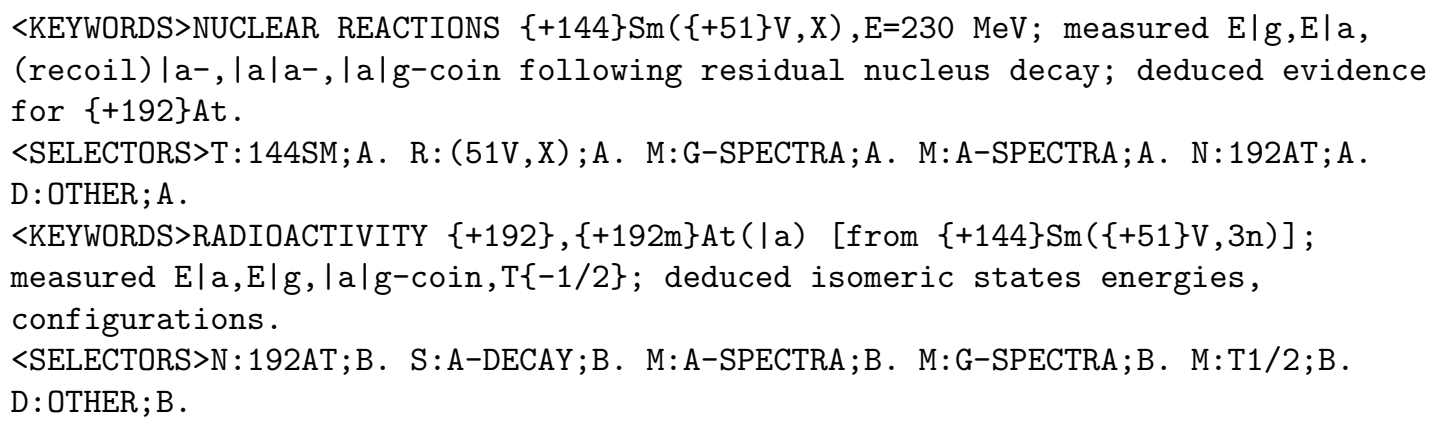

The "deduced evidence for" wording is a special form which tells the processing program to look for a nuclide string $(\{+192\}$ At in this case, for which the "N:192AT;A." selector string is generated). 


\subsection{General Considerations and Conventions}

\section{Quantity type}

It is important that newly measured quantities be indexed as "measured" in the keywords. As a general rule, papers in which quantities are measured will have a section on the details of the experiment, and will include numerical information on measured quantities in tabular or graphical form.

The distinction between "calculated" and "analyzed" is not always clear. As a rule, if quantities are calculated using a pre-existing set of parameters and then compared with data, then using "calculated" is appropriate. If the main thrust of the paper is to fit a set of data in order to obtain model parameters, then "analyzed" might be a better choice. To some extent, the author's choice of words can be used as a guide.

As mentioned earlier, it is expected that "deduced" phrases will not appear alone in a keyword abstract, but will be preceded by a "measured", "calculated", or "analyzed" phrase.

\section{Selector generation}

Searches on the indexed selectors constitute a large fraction of NSR usage. Because of this, the person preparing keywords should try to ensure that all selectors relevant to a given paper are in fact generated and present in the selector field.

Often, this is simply a case of including all important quantities discussed in a paper. For instance, including "E-g" will generate "G-SPECTRA", "J" or "-p" will generate "LEVEL-PROP", and "- d" (mixing ratio) will generate "G-MULTIPOLARITY".

In some cases, it is important to phrase the keywords correctly in order to get all of the desired selectors. For example, "- a spectrum" and "proton spectrum" will individually generate "A-SPECTRA" and "P-SPECTRA", but the phrase "proton and - a spectra" will only generate "A-SPECTRA". In this case, it might be better to use "Ep and E- a".

In other cases, it is important to keep in mind that certain symbols might generate different selectors than expected. For instance, "Q" by itself generates "Q-VALUES", so the word "quadrupole" should be spelled out to generate the "QUADRUPOLE" selector.

Sometimes, an expected selector may not appear. For example, a neutron- 
induced fission reaction is expressed as " $(\mathrm{n}, \mathrm{F})$ " in NSR. This will produce an "(N,F)" reaction selector, but will not by itself generate the "FISSION" selector. For that, the word "FISSION" should appear in the quantity phrase (i.e. "measured fission fragment spectra").

\section{Excessively long lists}

Often, compilations or theory papers will publish tables and figures which include information on a large number of nuclides. Listing all of these nuclides could in principle cause problems. This is because the most common type of search on NSR is for information for a given nuclide. If every nuclide search were to retrieve the same set of references, it would make the database less useful. For papers that list information for more than about 100 nuclides, it is preferable to give an atomic mass and/or number range such as " $\mathrm{A}=4-208$ " or " $\mathrm{Z}=2-82$ ".

\section{Intermediate quantities}

As a rule, it is best to include "measured" quantities when there is some numerical or graphical expression of the quantity in the paper. For example, in activation experiments, the authors generally measure gamma spectra in

order to determine reaction product yields. However, the spectra themselves are generally not shown, and the correct measured quantity to include in the keywords is yield, cross section, or excitation function. For papers where gamma transition energies are used to establish level schemes, on the other hand, sample spectra are usually shown and it is appropriate to include "measured E-g".

\section{Review articles}

It is often the case that a review article will include small pieces of information on a large number of systems. In general, these papers are not given keyword abstracts, since the quantities discussed have presumably already appeared in the literature. In a few cases, where the contents of the paper cover only one or a few systems and can be summarized concisely, then keywords may be prepared. 


\section{Other considerations}

In order to be useful, the keyword abstracts should be reasonably concise. Depending on the type of research being reported, some papers lend themselves to brief summarization, and some do not. Papers discussing complex nuclear reactions often end with a complex set of conclusions. In this case it is often best to summarize the keyword abstract with the phrase "deduced reaction mechanism features".

Whether to describe a set of levels as "high-spin" (which generates the selector "HIGH-SPN") can often be decided by following the authors' language. In order to limit redundancy, keyword abstracts have not, as a rule, included "rotational bands" (selector "ROT-BANDS") in cases where "highspin levels" are part of the keywords. 


\section{Chapter 4}

\section{Scope and Completeness}

As the name implies, NSR is intended to cover all nuclear science literature in which new nuclear properties are discussed. Originally "Nuclear Structure References", the name was changed in the mid 1990's to reflect an expanded scope. Since that time, all papers from three journals are included: Physical Review C, Nuclear Physics A, and European Physical Journal A (formerly Zeitschrift für Physik A). The scope of NSR is roughly the same as the scope of these journals. It does not generally cover literature concerned with nuclear applications, such as neutron activation analysis for material characterization, or radiological dating techniques in archaeology. A list of journals covered regularly in NSR is given in appendix A and on the NSR web site at NNDC. Over 80 journals are checked on a regular basis for articles of interest.

Peer-reviewed journals are considered to be "primary" references in NSR, and an effort is made to be complete in the coverage of this literature. Articles of interest from "secondary" references such as laboratory reports and conference proceedings are also included. In order to try to avoid duplication, and to make most efficient use of the compilation effort, the choice of which articles to include from secondary references is more selective, with previously unpublished experimental results having the highest priority. It should be noted that conference proceedings published as journal issues are treated as primary references. Since the beginning of the year 2000, roughly $88 \%$ of the articles entered into NSR are from primary sources. 


\section{Appendix A}

\section{Journals and Codens}

The following list, arranged alphabetically by title, shows journal codens and titles that have appeared in NSR since the year 2000. Beginning in 2006, we are coding some translation journals rather than the original Russian, due to availability (i.e. PANUE rather than YAFIA). A more complete list, including all codens used in NSR, can be found at the NSR web site (http://www.nndc.bnl.gov/nsr/coden_dict.jsp).

$\begin{array}{lll}\text { APHPF Acta Phys.Hung.N.S. } & \text { CJPHA Can.J.Phys. } \\ \text { APOBB Acta Phys.Pol. B } & \text { CMPHC Chem.Phys. } \\ \text { APSVC Acta Phys.Slovaca } & \text { CHPLB Chem.Phys.Lett. } \\ \text { ACIEA Angew.Chem., Int.Ed.Eng. } & \text { CJOPA Chin.J.Phys.(Taiwan) } \\ \text { ANEND Ann.Nucl.Energy } & \text { CPLEE Chin.Phys.Lett. } \\ \text { ANPYA Ann.Phys.(Leipzig) } & \text { CHPHD Chinese Physics } \\ \text { APNYA Ann.Phys.(New York) } & \text { CPHCB Comput.Phys.Commun. } \\ \text { ANPHA Ann.Phys.(Paris) } & \text { CZYPA Czech.J.Phys. } \\ \text { ARNUA Ann.Rev.Nucl.Part.Sci. } & \text { DANKA Dok.Akad.Nauk } \\ \text { ARISE Appl.Radiat.Isot. } & \text { EPSLA Earth Planet.Sci.Lett. } \\ \text { APHYE Astropart.Phys. } & \text { ZAANE Eur.Phys.J. A } \\ \text { ASJOA Astrophys.J. } & \text { ZBBNE Eur.Phys.J. B } \\ \text { APJSA Astrophys.J.Suppl.Ser. } & \text { ZCCNE Eur.Phys.J. C } \\ \text { ADNDA At.Data Nucl.Data Tables } & \text { ZDDNE Eur.Phys.J. D } \\ \text { AENGA At.Energ. } & \text { EULEE Europhys.Lett. } \\ \text { AUJPA Aust.J.Phys. } & \text { FBSYE Few-Body Systems } \\ \text { BJPHE Braz.J.Phys. } & \text { FECAA Fiz.Elem.Chastits At.Yadra } \\ \text { BAPSA Bull.Am.Phys.Soc. } & \text { FIZBE Fizika(Zagreb) B } \\ \text { BRSPE Bull.Rus.Acad.Sci.Phys. } & \text { GCACA Geochim.Cosmochim.Act. }\end{array}$




$\begin{array}{llll}\text { HYIND } & \text { Hyperfine Interactions } & \text { PLRAA Phys.Rev. A } \\ \text { IMPEE } & \text { Int.J.Mod.Phys. E } & \text { PRBMD Phys.Rev. B } \\ \text { IJTPB } & \text { Int.J.Theor.Phys. } & \text { PRVCA Phys.Rev. C } \\ \text { KPSJA } & \text { J.Korean Phys.Soc. } & \text { PRVDA Phys.Rev. D } \\ \text { JNRSA } & \text { J.Nucl.Radiochem.Sci. } & \text { PLEEE Phys.Rev. E } \\ \text { JNSTA } & \text { J.Nucl.Sci.Technol.(Tokyo) } & \text { PRLTA Phys.Rev.Lett. } \\ \text { JPAMA } & \text { J.Phys.(London) B } & \text { PHSTB Phys.Scr. } \\ \text { JPGPE } & \text { J.Phys.(London) G } & \text { PHYAD Physica A } \\ \text { JCOME } & \text { J.Phys.Condens.Matter } & \text { PHYBE Physica B } \\ \text { JUPSA } & \text { J.Phys.Soc.Jpn. } & \text { PHYCE Physica C } \\ \text { JRNCD J.Radioanal.Nucl.Chem. } & \text { PZETA Pisma Zh.Eksp.Teor.Fiz. } \\ \text { JRNBA } & \text { J.Res.Natl.Inst.Stand.Technol. PRAMC Pramana } \\ \text { LAPLA } & \text { Laser Phys.Lett. } & \text { PNEND Prog.Nucl.Energy } \\ \text { MPLAE } & \text { Mod.Phys.Lett. A } & \text { PPNPD Prog.Part.Nucl.Phys. } \\ \text { NATUA } & \text { Nature(London) } & \text { PTPKA Prog.Theor.Phys.(Kyoto) } \\ \text { NJOPF } & \text { New Journal of Physics } & \text { PTPSA Prog.Theor.Phys.(Kyoto), Suppl. } \\ \text { NDSBA } & \text { Nucl.Data Sheets } & \text { PACHA Pure Appl.Chem. } \\ \text { NIMAE } & \text { Nucl.Inst.Meth.Phys.Res. A } & \text { RMEAE Radiat.Meas. } \\ \text { NIMBE } & \text { Nucl.Inst.Meth.Phys.Res. B } & \text { RAACA } & \text { Radiochim.Acta } \\ \text { NUPAB } & \text { Nucl.Phys. A } & \text { RPPHA Rep.Prog.Phys. } \\ \text { NUPBB Nucl.Phys. B } & \text { RMPHA Rev.Mod.Phys. } \\ \text { NPBSE Nucl.Phys. B(Proc.Supp.) } & \text { RNCUA Riv.Nuovo Cimento Soc.Ital.Fis. } \\ \text { NSENA Nucl.Sci.Eng. } & \text { RJPHE Rom.J.Phys. } \\ \text { NUTYB Nucl.Technology } & \text { SCIEA Science } \\ \text { NIFBA Nuovo Cim. B } & \text { TANSA Trans.Amer.Nucl.Soc. } \\ \text { NIFCA Nuovo Cim. C } & \text { TBGNS Trans.Bulg.Nucl.Soc. } \\ \text { FECLA } & \text { Part. and Nucl., Lett. } & \text { TJPHE Turk.J.Phys. } \\ \text { PANUE Phys.Atomic Nuclei } & \text { UKPJA Ukr.J.Phys. } \\ \text { PYLAA } & \text { Phys.Lett. A } & \text { YAFIA Yad.Fiz. } \\ \text { PYLBB } & \text { Phys.Lett. B } & \text { ZNASE Z.Naturforsch. } \\ \text { PRPLC Phys.Rep. } & \text { ZETFA Zh.Eksp.Teor.Fiz. } \\ & \end{array}$




\section{Appendix B}

\section{Abbreviations}

Because there is a varied audience for NSR, the use of abbreviations that might only be familiar to specialists should be avoided. For example, "timedependent perturbed angular distribution" is typically spelled out in the keywords, rather than using "TDPAD".

The following abbreviations are commonly used in NSR. In some cases, specific abbreviations are recognized by the selector generation program; these are noted.

$\begin{array}{ll}\text { Ay,Ayy,iT } \ldots & \text { - analyzing powers }(1) \\ \text { BCS } & \text { - Bardeen-Cooper-Schieffer } \\ \text { BUU } & \text { - Boltzmann-Uehling-Uhlenbeck } \\ \text { ce } & \text { - conversion electron (2) } \\ \text { CPT } & \text { - charge-parity-time } \\ \text { DSA } & \text { - Doppler-shift attenuation (3) } \\ \text { DWBA } & \text { - distorted-wave Born approximation } \\ \text { DWIA } & \text { - distorted-wave impulse approximation } \\ \text { E } \gamma, \text { E } \alpha, \text { Ep } & \text { - gamma, alpha, proton energies (4) } \\ \text { EC } & \text { - electron capture } \\ \text { GMR } & \text { - giant monopole resonance } \\ \text { GDR } & \text { - giant dipole resonance (5) } \\ \text { GQR } & \text { - giant quadrupole resonance (5) } \\ \text { GDH } & \text { - Gerasimov-Drell-Hearn } \\ \text { HFB } & \text { - Hartree-Fock-Bogoliubov } \\ \text { hfs } & \text { - hyperfine structure } \\ \text { I } \gamma, \text { I } \beta & \text { - gamma, beta intensities (4) } \\ \text { IAR } & \text { - isobaric analog resonance (6) }\end{array}$




$\begin{array}{ll}\text { IAS } & \text { - isobaric analog state }(6) \\ \text { IBM } & \text { - interacting boson model } \\ \text { IBA } & \text { - interacting boson approximation } \\ \text { ICC } & \text { - internal conversion coefficient }(2) \\ \text { IT } & \text { - isomeric transition } \\ \text { H } & \text { - magnetic field } \\ \text { n-bar, p-bar } & \text { - antineutron, antiproton } \\ \text { OZI } & \text { - Okubo-Zweig-Iizuka } \\ \text { PWIA } & \text { - plane-wave impulse approximation } \\ \text { Q } \alpha, \text { Q } \beta & \text { - } \alpha \text {-, } \beta \text {-decay Q-value }(7) \\ \text { QCD } & \text { - quantum chromodynamics } \\ \text { RPA } & \text { - random phase approximation } \\ \sigma & \text { - cross section }(8) \\ \text { SUSY } & \text { - supersymmetry } \\ \theta & \text { - indicates angular dependence } \\ \text { TDA } & \text { - Tamm-Dancoff approximation }\end{array}$

Notes:

(1) - generates selector: POLARIZATION

(2) - generates selector: $\mathrm{CE}$

(3) - generates selector: DOPPLER

(4) - depending on particles, generates selectors G-SPECTRA, P-SPECTRA ...

(5) - generates selector: RESONANCE

(6) - generates selector: ANALOGS

(7) - generates selector: Q

(8) - generates selector SIGMA if by itself, DSIGMA if followed by variables in parentheses 


\section{Appendix $\mathrm{C}$}

\section{Special Characters}

Superscripting and subscripting in NSR are done using the form " $\{+\mathrm{xxx}\}$ " and " $\{-\mathrm{xxx}\}$ " where "xxx" is the text to be superscripted or subscripted. Note that these cannot be nested (i.e. subscript on a subscript, etc.).

"Overline"-type characters are not currently recognized in NSR. In order to express, for example, $\vec{p}$ or $\bar{p}$, use " $\mathrm{p}(\mathrm{pol})$ " and "p-bar".

Special characters such as greek symbols are expressed in NSR by placing a "|" character before a regular character. The table below gives the various translations. Preceding the character in the first column with a "|" will produce the symbol in the second column. For example, to put the symbol $\gamma$ into a text field, use "Ig". The third, fourth, and fifth columns show how the character will be translated to Text, HTML, and LaTex formats. Note that the LaTex expression will generally be enclosed in a math environment.

Special characters in NSR.

\begin{tabular}{lllll} 
Character & Symbol & Text & HTML & LaTex \\
\hline $\mathrm{A}$ & $A$ & ALPHA & \&Alpha; & $\mathrm{A}$ \\
$\mathrm{B}$ & $B$ & BETA & \&Beta; & $\mathrm{B}$ \\
$\mathrm{C}$ & $H$ & ETA & \&Eta; & $\mathrm{H}$ \\
$\mathrm{D}$ & $\Delta$ & DELTA & \&Delta; & $\backslash$ Delta \\
$\mathrm{E}$ & $E$ & EPSILON & \&Epsilon; & $\mathrm{E}$ \\
$\mathrm{F}$ & $\Phi$ & PHI & \&Phi; & $\backslash$ Phi \\
$\mathrm{G}$ & $\Gamma$ & GAMMA & \&Gamma; & $\backslash$ Gamma \\
$\mathrm{H}$ & $X$ & CHI & \&Chi; & X \\
$\mathrm{I}$ & $I$ & IOTA & \&Iota; & I \\
\hline & & & \multicolumn{2}{c}{ Continued on next page }
\end{tabular}


Special characters in NSR, continued

\begin{tabular}{|c|c|c|c|c|}
\hline Character & Symbol & Text & HTML & LaTex \\
\hline $\mathrm{J}$ & $\sim$ & & \&sim; & $\backslash \operatorname{sim}$ \\
\hline K & $K$ & KAPPA & \&Kappa; & $\mathrm{K}$ \\
\hline $\mathrm{L}$ & $\Lambda$ & LAMBDA & \&Lambda; & $\backslash$ Lambda \\
\hline M & $M$ & MU & $\& \mathrm{Mu}$ & M \\
\hline $\mathrm{N}$ & $N$ & $\mathrm{NU}$ & $\& N u$ & $\mathrm{~N}$ \\
\hline $\mathrm{O}$ & $O$ & $\mathrm{O}$ & \&Omicron; & $\mathrm{O}$ \\
\hline $\mathrm{P}$ & $\Pi$ & PI & $\& \mathrm{Pi}$ & $\backslash \mathrm{Pi}$ \\
\hline Q & $\Theta$ & THETA & \&Theta; & \Theta \\
\hline $\mathrm{R}$ & $R$ & $\mathrm{RHO}$ & \&Rho; & $\mathrm{R}$ \\
\hline S & $\Sigma$ & SIGMA & \&Sigma; & $\backslash$ Sigma \\
\hline $\mathrm{T}$ & $T$ & TAU & $\& T a u$ & $\mathrm{~T}$ \\
\hline $\mathrm{U}$ & $\Upsilon$ & UPSILON & \&Upsilon; & $\backslash$ Upsilon \\
\hline V & $\nabla$ & PARTIAL & \&nabla; & \nabla \\
\hline W & $\Omega$ & OMEGA & \&Omega; & $\backslash$ Omega \\
\hline $\mathrm{X}$ & $\Xi$ & XI & $\& \mathrm{Xi}$ & $\backslash \mathrm{Xi}$ \\
\hline $\mathrm{Y}$ & $\Psi$ & PSI & \&Psi; & $\backslash$ Psi \\
\hline Z & $Z$ & ZETA & \&Zeta; & $\mathrm{Z}$ \\
\hline $\mathrm{a}$ & $\alpha$ & alpha & \&alpha; & $\backslash$ alpha \\
\hline $\mathrm{b}$ & $\beta$ & beta & \&beta; & $\backslash$ beta \\
\hline $\mathrm{c}$ & $\eta$ & eta & \&eta; & $\backslash$ eta \\
\hline d & $\delta$ & delta & \&delta; & $\backslash$ delta \\
\hline e & $\varepsilon$ & epsilon & \&\#1013; & \varepsilon \\
\hline $\mathrm{f}$ & $\phi$ & phi & \&phi; & $\backslash$ phi \\
\hline$g$ & $\gamma$ & gamma & \&gamma; & Igamma \\
\hline h & $\chi$ & chi & \&chi; & $\backslash$ chi \\
\hline i & $\iota$ & iota & \&iota; & $\backslash$ iota \\
\hline j & $\epsilon$ & vrepsilon & \&epsilon; & \epsilon \\
\hline $\mathrm{k}$ & $\kappa$ & kappa & \&kappa; & $\backslash$ kappa \\
\hline 1 & $\lambda$ & lambda & \&lambda; & $\backslash$ lambda \\
\hline $\mathrm{m}$ & $\mu$ & $\mathrm{mu}$ & $\& m u$ & $\backslash \mathrm{mu}$ \\
\hline $\mathrm{n}$ & $\nu$ & nu & \&nu; & $\backslash n u$ \\
\hline $\mathrm{O}$ & $o$ & $\mathrm{O}$ & \&omicron; & o \\
\hline $\mathrm{p}$ & $\pi$ & pi & \&pi; & $\backslash$ pi \\
\hline q & $\theta$ & theta & \&theta; & $\backslash$ theta \\
\hline $\mathrm{r}$ & $\rho$ & rho & \&rho; & $\backslash$ rho \\
\hline $\mathrm{s}$ & $\sigma$ & sigma & \&sigma; & \sigma \\
\hline
\end{tabular}


Special characters in NSR, continued

\begin{tabular}{|c|c|c|c|c|}
\hline Character & Symbol & Text & HTML & LaTex \\
\hline $\mathrm{t}$ & $\tau$ & tau & \&tau; & $\backslash$ tau \\
\hline $\mathrm{u}$ & $v$ & upsilon & \&upsilon; & \upsilon \\
\hline $\mathrm{w}$ & $\omega$ & omega & \&omega; & \omega \\
\hline $\mathrm{x}$ & $\xi$ & $\mathrm{xi}$ & $\& x i$ & $\backslash x i$ \\
\hline $\mathrm{y}$ & $\psi$ & psi & \&psi; & $\backslash p s i$ \\
\hline $\mathrm{Z}$ & $\zeta$ & zeta & \&zeta; & $\backslash$ zeta \\
\hline , & $\circ$ & degrees & \&deg; & $\backslash$ circ \\
\hline ‘ & $\ell$ & scriptl & $\& \# 8467$ & $\backslash$ ell \\
\hline( & $\leftarrow$ & FROM & \&larr; & $\backslash$ gets \\
\hline ) & $\rightarrow$ & TO & \&rarr; & $\backslash$ to \\
\hline$*$ & $\times$ & $\mathrm{x}$ & \&times; & $\backslash$ times \\
\hline+ & \pm & $\mathrm{PM}$ & \&plusmn & $\backslash \mathrm{pm}$ \\
\hline- & $\mp$ & $\mathrm{MP}$ & $\& \# 8723$ & $\backslash \mathrm{mp}$ \\
\hline & $\propto$ & PROPTO & \&prop; & $\backslash$ propto \\
\hline 4 & $<$ & $<$ & \&lt; & $<$ \\
\hline 5 & $>$ & $>$ & \&gt; & $>$ \\
\hline 6 & $\sqrt{ }$ & SQRT & \&radic; & $\backslash$ surd \\
\hline 7 & $\int$ & INTEGRAL & \&int; & $\backslash$ int \\
\hline 8 & $\prod$ & PRODUCTOF & \&prod; & $\backslash$ prod \\
\hline 9 & $\sum$ & SUMOF & \&sum; & $\backslash$ sum \\
\hline$<$ & $\leq$ & $\mathrm{LE}$ & \&le; & $\backslash \mathrm{le}$ \\
\hline$=$ & $\neq$ & $\mathrm{NOT}=$ & \&ne; & $\backslash$ ne \\
\hline$>$ & $\geq$ & GE & \&ge; & $\backslash$ ge \\
\hline$?$ & $\approx$ & $\mathrm{AP}=$ & \&asymp; & \approx \\
\hline @ & $\infty$ & INFINITY & \&infin; & $\backslash$ infty \\
\hline[ & \{ & \{ & & \\
\hline 1 & ? & ! & ! & \\
\hline ] & $\begin{array}{l}\} \\
\uparrow\end{array}$ & UPARROW & $\begin{array}{l}\text { \} } \\
\text { \&uarr; }\end{array}$ & If uparrow \\
\hline - & $\downarrow$ & DOWNARROW & \&darr; & \downarrow \\
\hline
\end{tabular}

